\title{
ON SMALL SETS OF DISTRIBUTION FUNCTIONS OF RATIO BLOCK SEQUENCES
}

\author{
DaVid KrČMarskÝ — LAdislaV MišíK — ZuZana VÁClavíKová \\ Dedicated to the memory of Professor Pierre Liardet
}

\begin{abstract}
There are various methods how to describe and characterize distribution of elements of sets of positive integers. One of the most interesting is that using the set of all distribution functions of the corresponding ratio block sequence introduced in [Strauch, O.—Tóth, J. T.: Publ. Math. Debrecen 58 (2001), no. 4, 751-778]. In the present paper we give some sufficient conditions under which this set is small in a metric sense. As a corollary we obtain a new characterization of the case of asymptotic distribution.
\end{abstract}

\section{Communicated by Oto Strauch.}

\section{Introduction}

\section{Basic definitions and properties}

- From now on $X=\left\{x_{1}<x_{2}<\cdots\right\}$ denotes an increasing sequence of positive integers and $x \in[0,1)$.

- For every positive integer $n$ put

$$
X_{n}=\left(\frac{x_{1}}{x_{n}}, \frac{x_{2}}{x_{n}}, \cdots \frac{x_{n}}{x_{n}}\right)
$$

the $n$th term of the ratio block sequence $\left(X_{n}\right)_{n=1}^{\infty}$.

- Denote by $F\left(X_{n}, x\right)$ the step distribution function

$$
F\left(X_{n}, x\right)=\frac{\#\left\{i \leq n ; \frac{x_{i}}{x_{n}}<x\right\}}{n},
$$

for $x \in[0,1)$ and for $x=1$ we define $F\left(X_{n}, 1\right)=1$.

2010 Mathematics Subject Classification: 11B05.

Keyw ords: asymptotic distribution function, uniform distribution, block sequence.

This work was supported by the project P201/12/2351 of GAČR. 


\section{DAVID KRČMARSKÝ — LADISLAV MIŠÍK — ZUZANA VÁCLAVÍKOVÁ}

- Directly from the definition we obtain (see $[\mathrm{ST}]$ )

$$
F\left(X_{m}, x\right)=\frac{n}{m} F\left(X_{n}, x \frac{x_{m}}{x_{n}}\right)
$$

for each $m \leq n$ for every $x \in[0,1)$.

- For any positive $t$ denote by $X(t)$ the counting function

$$
X(t)=\#\left\{n \in \mathbb{N} ; x_{n}<t\right\} .
$$

The lower asymptotic density $\underline{d}$ and the upper asymptotic density $\bar{d}$ of $X=$ $\left\{x_{1}<x_{2}<\ldots\right\} \subset \mathbb{N}$ are defined as

$$
\begin{aligned}
& \underline{d}(X)=\liminf _{t \rightarrow \infty} \frac{X(t)}{t}=\liminf _{n \rightarrow \infty} \frac{n}{x_{n}}, \\
& \bar{d}(X)=\limsup _{t \rightarrow \infty} \frac{X(t)}{t}=\limsup _{n \rightarrow \infty} \frac{n}{x_{n}} .
\end{aligned}
$$

In the case when $\underline{d}(X)=\bar{d}(X)$ we speak about asymptotic density of $X$ and denote this common value by $d(X)$.

- A non-decreasing function

$$
g:[0,1] \rightarrow[0,1], \quad g(0)=0, \quad g(1)=1
$$

is called distribution function (abbreviating d.f.). We shall identify any two d.f. coinciding at common points of continuity. We will denote the set of all distribution functions by $\mathcal{D}$. Let us note that $\mathcal{D}$ endowed with the metric

$$
\varrho\left(g_{1}, g_{2}\right)=\sqrt{\int_{0}^{1}\left(g_{1}(x)-g_{2}(x)\right)^{2} \mathrm{~d} x}
$$

is a compact topological space [W].

- A d.f. $g(x)$ is a d.f. of the sequence of blocks $\left(X_{n}\right)_{n=1}^{\infty}$ if there exists an increasing sequence $n_{1}<n_{2}<\cdots$ of positive integers such that

$$
\lim _{k \rightarrow \infty} F\left(X_{n_{k}}, x\right)=g(x) \quad \text { a.e. on }[0,1] \text {. }
$$

This is equivalent to the weak convergence, i.e., the preceding limit holding for every point $x \in[0,1]$ of continuity of $g(x)$, and it is also equivalent to the convergence with respect to the $\mathcal{L}^{2}$ metric in $\mathcal{D}$.

- Denote by $G\left(X_{n}\right)$ the set of all d.f.s of a ratio block sequence $\left(X_{n}\right)$. For a singleton $G\left(X_{n}\right)=\{g(x)\}$, the d.f. $g(x)$ is also called asymptotic d.f. (abbreviating a.d.f.) of $\left(X_{n}\right)$. 


\section{ON SMALL SETS OF DISTRIBUTION FUNCTIONS OF RATIO BLOCK SEQUENCES}

- It is evident that every $G\left(X_{n}\right)$ is nonempty, by compactness of $\mathcal{D}$, and closed, as a set of all limit points of a sequence in metric space. One of the most challenging open problem of theory of sets of distribution functions of ratio block sequences is that of characterization of all possible sets $G\left(X_{n}\right)$, see [SN]. It can be formulated as: given a nonempty closed subset $H$ of $\mathcal{D}$, decide if there exists a set $X \subset \mathbb{N}$ such that $G\left(X_{n}\right)=H$. The simplest case, when $G\left(X_{n}\right)$ is a singleton was characterized in $[\mathrm{ST}]$. It was shown that the only possible singletons are of the form $G\left(X_{n}\right)=\left\{x^{\lambda}\right\}$, where $\lambda \in[0,1]$ (here $x^{0}$ means the distribution function identically equal to 1 for all $x \in(0,1])$. In addition, the case $\lambda=0$ was completely characterized. The remaining cases for $\lambda \in(0,1]$ were characterized in [FT], where it is shown that for $0<\lambda \leq 1$ the equality $G\left(X_{n}\right)=\left\{x^{\lambda}\right\}$ happens if and only if

$$
\lim _{n \rightarrow \infty} \frac{x_{k n}}{x_{n}}=k^{\frac{1}{\lambda}} \quad \text { holds for every } k=1,2, \ldots
$$

For more information in the topic see [SP], [ST] and [GS].

- In this paper, we will show that if there exists a function $f \in G\left(X_{n}\right)$ and a sequence of indices $\left(n_{k}\right)$ such that $f=\lim _{k \rightarrow \infty} F\left(X_{n_{k}}, x\right)$ and $\left(n_{k}\right)$ is large in some sense, then the set $G\left(X_{n}\right)$, as a subset of the metric space $(\mathcal{D}, \varrho)$, has a small diameter.

As corollaries of the general theorem we give several sufficient conditions under which the set $G\left(X_{n}\right)$ is a singleton. We will show that if there exists a function $f \in G\left(X_{n}\right)$ such that the set of indices $n_{k}$ with $f=\lim _{k \rightarrow \infty} F\left(X_{n_{k}}, x\right)$ is sufficiently large then necessarily $G\left(X_{n}\right)=\{f\}$. Usually the term "sufficiently large set" is related to asymptotic density. We will also use another kind of density, the so called gap density introduced in the paper [GV]. It is defined by

$$
\lambda(X)=\limsup _{n \rightarrow \infty} \frac{x_{n+1}}{x_{n}}, \quad \text { where } \quad X=\left\{x_{1}<x_{2}, \ldots\right\} \subset \mathbb{N} .
$$

\section{Results}

The following simple lemma plays the key role in our investigation.

Lemma 1. Let $m<n$ be positive integers. Then

$$
F\left(X_{n}, x\right) \geq \frac{m}{n} F\left(X_{m}, x\right)
$$

for every $x \in[0,1]$. 


\section{DAVID KRČMARSKÝ — LADISLAV MIŠÍK - ZUZANA VÁCLAVÍKOVÁ}

P r o of. Let $m<n$ be positive integers and $x \in(0,1)$. Using the obvious inclusion

$$
\left\{i \leq m ; \frac{x_{i}}{x_{m}}<x\right\} \subset\left\{i \leq n ; \frac{x_{i}}{x_{n}}<x\right\}
$$

calculate

$$
F\left(X_{n}, x\right)=\frac{\#\left\{i \leq n ; \frac{x_{i}}{x_{n}}<x\right\}}{n} \geq \frac{\#\left\{i \leq m ; \frac{x_{i}}{x_{m}}<x\right\}}{m} \frac{m}{n}=\frac{m}{n} F\left(X_{m}, x\right) .
$$

Recall that the diameter of a set $A$, a subset of a metric space $(X, \varrho)$, is defined by

$$
\operatorname{diam} A=\sup \left\{\varrho\left(a, a^{\prime}\right) \mid a, a^{\prime} \in A\right\} .
$$

Now we are prepared to formulate our general theorem.

Theorem 1. Let $X \subset \mathbb{N}, f \in G\left(X_{n}\right)$ and let $J=\left\{j_{1}<j_{2}<\ldots\right\} \subset \mathbb{N}$ be such that

$$
\lim _{j \in J} F\left(X_{j}, x\right)=f(x) \quad \text { and } \quad \lambda(J)<\infty
$$

Then

$$
\operatorname{diam} G\left(X_{n}\right) \leq(\lambda(J)-1) \sqrt{\int_{0}^{1} f^{2}(x) \mathrm{d} x} .
$$

P r o of. Put $\lambda=\lambda(J)$ and let $g$ be any function belonging to $G\left(X_{n}\right)$. A simple compactness argument yields that there exist:

- numbers $\alpha$ and $\beta$ such that $0<\frac{1}{\lambda} \leq \alpha \leq \beta \leq 1$,

- a subsequence $\left(u_{k}\right)$ of the sequence $\left(j_{n}\right)$ and

- a sequence of positive integers $\left(v_{k}\right)$ with $u_{k}<v_{k}<u_{k+1}$ for all $k \in \mathbb{N}$

suh that

$$
\lim _{k \rightarrow \infty} \frac{u_{k}}{u_{k+1}}=\alpha, \quad \lim _{k \rightarrow \infty} \frac{u_{k}}{v_{k}}=\beta \quad \text { and } \quad \lim _{k \rightarrow \infty} F\left(X_{v_{k}}, x\right)=g(x) .
$$

Two applications of Lemma 1 the first one with $m=u_{k}, n=v_{k}$ and the next one with $m=v_{k}, n=u_{k+1}$, yield

$$
F\left(X_{v_{k}}, x\right) \geq \frac{u_{k}}{v_{k}} F\left(X_{u_{k}}, x\right) \text { and } F\left(X_{u_{k+1}}, x\right) \geq \frac{v_{k}}{u_{k+1}} F\left(X_{v_{k}}, x\right),
$$

hence

$$
\frac{u_{k}}{v_{k}} F\left(X_{u_{k}}, x\right) \leq F\left(X_{v_{k}}, x\right) \leq \frac{u_{k+1}}{v_{k}} F\left(X_{u_{k+1}}, x\right) \quad \text { for all } \quad x \in[0,1] .
$$


Taking a limit in (5), by use of (44) we get

Consequently,

$$
\beta f(x) \leq g(x) \leq \frac{\beta}{\alpha} f(x) \text { for all } \quad x \in[0,1] .
$$

$$
\begin{aligned}
\operatorname{diam} G\left(X_{n}\right) & =\sup \left\{\varrho\left(g_{1}, g_{2}\right) \mid g_{1}, g_{2} \in G\left(X_{n}\right)\right\} \leq \varrho\left(\beta f(x), \frac{\beta}{\alpha} f(x)\right) \\
& =\sqrt{\int_{0}^{1}\left(\frac{\beta}{\alpha} f(x)-\beta f(x)\right)^{2} \mathrm{~d} x} \\
& =\sqrt{\left(\frac{\beta}{\alpha}-\beta\right)^{2} \int_{0}^{1} f^{2}(x) \mathrm{d} x} \leq(\lambda-1) \sqrt{\int_{0}^{1} f^{2}(x) \mathrm{d} x}
\end{aligned}
$$

where the last inequality follows from the fact that the function $\left(\frac{t}{\alpha}-t\right)^{2}$ attains its maximum $\left(\frac{1}{\alpha}-1\right)^{2} \leq(\lambda-1)^{2}$ in the interval $t \in[\alpha, 1]$ at point $t=1$.

It is evident, that the preceding theorem is valuable if either $\lambda(J)$ is not too much greater than 1 , or when the integral $\int_{0}^{1} f^{2}(x) \mathrm{d} x$ is small 1 . Two extremal cases, $\int_{0}^{1} f^{2}(x) \mathrm{d} x=0$ and $\lambda(J)=1$ will be discussed in the rest of the paper.

First, let us suppose that $\int_{0}^{1} f^{2}(x) \mathrm{d} x=0$. This means that $f=c_{1}$, where $c_{1}$ is the distribution function with total jump at 1 , i.e., $c_{1}(x)=0$ for all $x \in[0,1)$. The following statement says that if $c_{1}$ belongs to $G\left(X_{n}\right)$, then the corresponding subsequence of step distribution functions converging to $c_{1}$ has arbitrary large gaps.

Proposition 1. Let $X \subset \mathbb{N}$ and $J \subset \mathbb{N}$ be such that $\lim _{j \in J} F\left(X_{j}, x\right)=c_{1}(x)$. Then $\lambda(J)=\infty$.

P r o of. Suppose that $\lambda(J)<\infty$. Then, by Theorem 1, it would be that

$$
G\left(X_{n}\right)=\left\{c_{1}\right\} .
$$

But, by the result in $[\mathrm{ST}]$ characterising all possible singletons $G\left(X_{n}\right)$ and mentioned in the Introduction, this happens for no $X \subset \mathbb{N}$, a contradiction.

The extremal case $\lambda(J)=1$ is presented by the next theorem.

\footnotetext{
${ }^{1}$ Another possible kind of application of the previous theorem can be the following one. Suppose that we know $G\left(X_{n}\right)$, i.e., also $\operatorname{diam} G\left(X_{n}\right)$ for some $X \subset \mathbb{N}$. Then the inequality (3) provides a lower bound for $\lambda(J)$ for arbitrary $f(x) \in G\left(X_{n}\right)$, in special cases also for $f(x)=x$.
} 


\section{DAVID KRČMARSKÝ — LADISLAV MIŠÍK — ZUZANA VÁCLAVÍKOVÁ}

TheOREM 2. Let $X \subset \mathbb{N}$. Then $G\left(X_{n}\right)=\{f\}$ if and only if there exists a set $J \subset \mathbb{N}$ such that

$$
\lim _{j \in J} F\left(X_{j}, x\right)=f(x) \quad \text { and } \quad \lambda(J)=1 .
$$

Proof. The implication $\Rightarrow$ is evident as $\lim _{n \rightarrow \infty} F\left(X_{n}, x\right)=f(x)$. The opposite implication follows from Theorem 1 and the fact that for every subset $A$ of a metric space $\operatorname{diam} A=0$ if and only if $A$ is a singleton.

Example 1. Let $k \in \mathbb{N}$ and suppose that there exists $f \in G\left(X_{n}\right)$ such that $\lim _{n \rightarrow \infty} F\left(X_{n^{k}}, x\right)=f$. Then $G\left(X_{n}\right)=\{f\}$.

REMARK 1. Roughly spoken, Theorem 2 says that if there exists a distribution function in $G\left(X_{n}\right)$ so that it is a limit along a "quite large and regular" set of indices, then $G\left(X_{n}\right)$ is a singleton.

Now we will introduce two important special cases of the preceding theorem. For the first one we need the following definition. It was introduced by Pólya in $[\mathrm{Po}$ in a bit more general context.

Definition 1. Let $X \subset \mathbb{N}$. The lower and upper Pólya densities of $X$ are defined by

$$
\underline{p}(X)=\lim _{\delta \rightarrow 0^{+}} \liminf _{n \rightarrow \infty} \frac{\#(X \cap((1-\delta) n, n])}{\delta n}
$$

and

$$
\bar{p}(X)=\lim _{\delta \rightarrow 0^{+}} \limsup _{n \rightarrow \infty} \frac{\#(X \cap((1-\delta) n, n])}{\delta n},
$$

respectively.

REMARK 2. It is known $[\mathrm{PO}$ ] that for every set $X$ of positive integers the following inequalities

$$
0 \leq \underline{p}(X) \leq \underline{d}(X) \leq \bar{d}(X) \leq \bar{p}(X) \leq 1
$$

hold. In addition, the equality $\underline{d}(X)=\bar{d}(X)$ implies the equality $\underline{p}(X)=\bar{p}(X)$.

CoRollary 1. Let $X \subset \mathbb{N}$ be such that there exists a function $f \in G\left(X_{n}\right)$ and a set $K \subset \mathbb{N}$ with $\underline{p}(K)>0$ so that $\lim _{k \in K} F\left(X_{k}, x\right)=f$. Then $G\left(X_{n}\right)=\{f\}$.

Proof. Let $K=\left\{k_{1}<k_{2}<\cdots\right\}$. By Theorem 1 it is sufficient to prove that $\lim _{n \rightarrow \infty} \frac{k_{n+1}}{k_{n}}=1$. Suppose the contrary, i.e., $\lim _{\sup _{n \rightarrow \infty}} \frac{k_{n+1}}{k_{n}}=h>1$. Choose any $q \in\left(\frac{1}{h}, 1\right)$. Then there exists an increasing sequence $\left(n_{m}\right)$ of positive integers such that $\frac{k_{n_{m}-1}}{k_{n_{m}}}<q$. Put $\delta=1-q$ and notice that $K \cap\left((1-\delta) k_{n_{m}}, k_{n_{m}}\right]=\emptyset$ for every $m \in \mathbb{N}$, contradicting $\underline{p}(K)>0$. 


\section{ON SMALL SETS OF DISTRIBUTION FUNCTIONS OF RATIO BLOCK SEQUENCES}

The next statement follows from the previous one and the last sentence in Remark 2 ,

Corollary 2. Let $X \subset \mathbb{N}$ be such that there exists a function $f \in G\left(X_{n}\right)$ and a set $K \subset \mathbb{N}$ with $d(K)>0$ so that $\lim _{k \in K} F\left(X_{k}, x\right)=f$. Then $G\left(X_{n}\right)=\{f\}$.

In the previous corollary "quite large and regular" set of indices (see Remark 1) means that it possesses a positive asymptotic density. One can wonder what happens if we lose a part of this information, i.e., the set of indices is either "quite large" or "quite regular". The first case is discussed in Example 2. In fact, it says that there are very large sets of indices, with respect to asymptotic densities, such that the convergence along them does not guarantee that $G\left(X_{n}\right)$ is a singleton. To introduce the example we will need some notation and a simple lemma. For every $\gamma \in[0,1]$ let us denote by $h_{\gamma}$ the distribution function such that $h_{\gamma}(x)=\gamma$ for all $x \in(0,1)$.

Lemma 2. For every $n \in \mathbb{N}$ let a finite sequence $T_{n}=\left(t_{1}<t_{2}<\cdots<t_{k_{n}}\right)$ in $[0,1]$ be given and put $F_{n}(x)=\frac{\#\left\{i \leq k_{n} \mid t_{i}<x\right\}}{k_{n}}$. Suppose that there exists a sequence of non-negaitive integers $\left(m_{n}\right)$ with $m_{n} \leq k_{n}$ and such that

$$
t_{m_{n}} \rightarrow 0, \quad t_{m_{n}+1} \rightarrow 1 \quad \text { and } \quad \frac{m_{n}}{k_{n}} \rightarrow \gamma \in[0,1] .
$$

Then $F_{n}(x) \rightarrow h_{\gamma}(x)$ for every $x \in[0,1]$.

P r o of. The statement of lemma definitely holds for $x=0$ and $x=1$, thus suppose that $x \in(0,1)$. Then there exists $n_{0}$ such that for all $n>n_{0}$ inequalities $t_{m_{n}}<x<t_{m_{n}+1}$ hold. Thus

$$
F_{n}(x)=\frac{\#\left\{i \leq k_{n} \mid t_{i}<x\right\}}{k_{n}}=\frac{m_{n}}{k_{n}} \rightarrow \gamma .
$$

EXample 2. For every $\varepsilon \in(0,1)$ there exist sets $X \subset \mathbb{N}, K \subset \mathbb{N}$ such that

$$
\underline{d}(K)=1-\varepsilon, \quad \bar{d}(K)=1
$$

and $\lim _{k \in K} F\left(X_{k}, x\right)=h_{1}(x)$ for almost all $x \in[0,1]$, but

$$
G\left(X_{n}\right)=\left\{h_{\gamma} \mid \gamma \in[1-\varepsilon, 1]\right\},
$$

consequently $\operatorname{diam} G\left(X_{n}\right)=\varepsilon$.

Pr o of. First put $\eta=\frac{\varepsilon}{1-\varepsilon}$, i.e., $\varepsilon=\frac{\eta}{1+\eta}$. To construct the example set

$$
J=\bigcup_{n=1}^{\infty}[n !+2,(1+\eta) n !) \cap \mathbb{N} \text { and } K=\mathbb{N} \backslash J .
$$




\section{DAVID KRČMARSKÝ — LADISLAV MIŠÍK - ZUZANA VÁCLAVÍKOVÁ}

Then for every sufficiently large $n$

$$
J((1+\eta) n !) \approx \sum_{k=1}^{n} \eta k !=\eta \sum_{k=1}^{n} k ! \approx \eta n !
$$

and consequently,

$$
\underline{d}(K)=1-\bar{d}(J)=1-\lim _{n \rightarrow \infty} \frac{\eta n !}{(1+\eta) n !}=1-\frac{\eta}{1+\eta}=1-\varepsilon .
$$

Similarly,

$$
\bar{d}(K)=\lim _{n \rightarrow \infty} \frac{K(n !)}{n !}=\lim _{n \rightarrow \infty} \frac{n !-\eta(n-1) !}{n !}=1
$$

which verifies (8). Now define

$$
x_{k}=k ! \text { for all } k \in K, \quad x_{j}=x_{j-1}+1 \quad \text { for all } j \in J
$$

and let

Notice that

$$
X=\left\{x_{1}, x_{2}, \ldots\right\}
$$

$$
x_{n} \leq n \text { ! holds for every } n \in \mathbb{N} .
$$

Now we show that

$$
\lim _{k \in K} F\left(X_{k}, x\right)=h_{1}(x) \text { for all } \quad x \in[0,1] .
$$

Let $\left(k_{n}\right)$ be an increasing sequence in $K$. Then, by (91), for every $n$ all terms of the block

$$
X_{k_{n}}=\left(\frac{x_{1}}{x_{k_{n}}}, \frac{x_{2}}{x_{k_{n}}}, \cdots, \frac{x_{k_{n}-1}}{x_{k_{n}}}, \frac{x_{k_{n}}}{x_{k_{n}}}\right),
$$

except the last one are less than or equal to $\frac{x_{k_{n}-1}}{x_{k_{n}}} \leq \frac{\left(k_{n}-1\right) !}{\left(k_{n}\right) !} \leq \frac{1}{k_{n}} \rightarrow 0$. Using Lemma 2 with $m_{n}=k_{n}-1$, giving $\gamma=\lim _{n \rightarrow \infty} \frac{m_{n}}{k_{n}}=1$ yields

$$
\lim _{k \in K} F\left(X_{k}, x\right)=h_{1}(x) \text { for all } x \in[0,1] .
$$

Now suppose that $\left(j_{n}\right)$ is an increasing sequence in $J$ such that there exists

$$
\lim _{n \rightarrow \infty} F\left(X_{j_{n}}, x\right) \text { for almost all } x \in[0,1] .
$$

Then, passing to a suitable subsequence, if necessary, we can assume that there exists an increasing sequence of positive integers $\left(k_{n}\right)$ such that

(i) $\left(k_{n}\right) !<j_{n} \leq(1+\eta)\left(k_{n}\right)$ !

(ii) $\lim _{n \rightarrow \infty} \frac{\left(k_{n}\right) !}{j_{n}}=\gamma \in\left[\frac{1}{1+\eta}, 1\right]$. 


\section{ON SMALL SETS OF DISTRIBUTION FUNCTIONS OF RATIO BLOCK SEQUENCES}

For simplicity put $K_{n}=\left(k_{n}\right)$ ! and look at the terms of

$$
X_{j_{n}}=\left(\frac{x_{1}}{x_{j_{n}}}, \cdots, \frac{x_{K_{n}}}{x_{j_{n}}}, \frac{x_{K_{n}+1}}{x_{j_{n}}}, \cdots, \frac{x_{j_{n}}}{x_{j_{n}}}\right) .
$$

By definition of sets $J$ and $K$, the elements $K_{n}$ and $K_{n}+1$ belong to $K$, while $K_{n}+2 \in J$. Consequently, for $m \leq K_{n}$ we have

$$
\frac{x_{m}}{x_{j_{n}}} \leq \frac{x_{K_{n}}}{x_{j_{n}}} \leq \frac{\left(K_{n}\right) !}{\left(K_{n}+1\right) !}=\frac{1}{K_{n}+1} \rightarrow 0 .
$$

On the other hand, for $K_{n}<m \leq j_{n}$ and putting

$$
m-K_{n}-1=a, \quad j_{n}-K_{n}-1=b,
$$

we have

$$
\frac{x_{m}}{x_{j_{n}}}=\frac{\left(K_{n}+1\right) !+a}{\left(K_{n}+1\right) !+b} \rightarrow 1
$$

as both $a$ and $b$ are less than or equal to

$$
\eta K_{n}=o\left(\left(K_{n}\right) !\right) \text {. }
$$

Now application of Lemma 2 yields

$$
\lim _{n \rightarrow \infty} F\left(X_{j_{n}}, x\right) \rightarrow h_{\gamma}(x) \text { for almost all } x \in[0,1] .
$$

On the other hand, for each $\gamma \in\left[\frac{1}{1+\eta}, 1\right]$ such a sequence $\left(j_{n}\right)$ exists, it is sufficient to put $j_{n}=\left\lfloor\frac{1}{\gamma} n !\right\rfloor$. This proves

$$
G\left(X_{n}\right)=\left\{h_{\gamma} \mid \gamma \in[1-\varepsilon, 1]\right\} \quad \text { and } \operatorname{diam} G\left(X_{n}\right)=\varepsilon .
$$

In the context of the previous example, let us note that in GS an example of an increasing sequence of positive integers $x_{n}, n=1,2, \ldots$ such that there is given

$$
G\left(x_{n}\right)=\left\{h_{\alpha}(x) ; \alpha \in[0,1]\right\}
$$

\section{REFERENCES}

[FT] FILIP, F.-TÓTH, J. T.: Characterization of asymptotic distribution functions of ratio block sequences, Periodica Mathematica Hungarica 60 (2010), no. 2, 115-126.

[GS] G: REKOS, G.-STRAUCH, O. Distribution functions of ratio sequences, II, Unif. Distrib. Theory 2 (2007), no. 1, 53-77.

[GV] GREKOS, G.-VOLKMANN, B.: On densities and gaps, Journal of Number Theory 26 (1987), 129-148.

[Po] PÓLYA, G.: Untersuchungen über Lücken und Singularitäten von Potenzreihen, Math. Zeit. 29 (1929), 549-640. 


\section{DAVID KRČMARSKÝ — LADISLAV MIŠÍK — ZUZANA VÁCLAVÍKOVÁ}

[SN] STRAUCH,O.-NAIR, R.: Unsolved Problems, http://udt.mat.savba.sk/, March 1, 2009.

[SP] STRAUCH, O.-PORUBSKÝ, Š.: Distribution of Sequences: A Sampler, Peter Lang, Frankfurt am Main, 2005.

[ST] STRAUCH, O.-TÓTH, J.T.: Distribution functions of ratio sequences, Publ. Math. Debrecen 58 (2001), no. 4, 751-778.

[W] WINKLER, R.: On the distribution behaviour of sequences, Math. Nachr. 186 (1997), 303-312.

Received January 14, 2016

Accepted April 7, 2016
David Krčmarský

Ladislav Mišík

Zuzana Václavíková

Department of Mathematics

University of Ostrava

30. dubna 22

70103 Ostrava 1

CZECH REPUBLIC

E-mail: ladislav.misik@osu.cz 